\title{
Nanoscale
}

D) Check for updates

Cite this: Nanoscale, 2018, 10, 16434

Received 16th May 2018,

Accepted 13th July 2018

DOI: $10.1039 / \mathrm{c} 8 \mathrm{nr} 03962 \mathrm{c}$

rsc.li/nanoscale

\section{How the crystal structure and phase segregation of Au-Fe alloy nanoparticles are ruled by the molar fraction and size $\uparrow$}

\author{
Anna Tymoczko, ${ }^{a}$ Marius Kamp, ${ }^{\text {b }}$ Oleg Prymak, ${ }^{\mathrm{c}}$ Christoph Rehbock, ${ }^{\text {J Jurij Jakobi, }}$ \\ Ulrich Schürmann, ${ }^{b}$ Lorenz Kienle ${ }^{b}$ and Stephan Barcikowski (iD *a
}

\begin{abstract}
The application of an Au-Fe nanoalloy is determined by its internal phase structure. Our experimental and theoretical findings explain how the prevalence of either a core-shell or a disordered solid solution structure is ruled by the target composition and the particle diameter. Furthermore, we found metastable phases not predefined by the bulk phase diagram.
\end{abstract}

Au-Fe nanomaterials with a defined internal structure ${ }^{1}$ are beneficial for numerous possible applications, ${ }^{2}$ e.g. for electrocatalytic oxygen evolution ${ }^{3}$ or during MRI/optical dual imaging. ${ }^{4}$ Furthermore, the Au-Fe nano-system is highly interesting from a fundamental point of view due to extended miscibility gaps and deviating crystal structures of its constituents in the bulk (Au-face centered cubic (FCC) vs. Fe-body-centered cubic (BCC)). ${ }^{5}$ To date it is basically unknown to what extent the internal phase and crystal structure of Au-Fe nanoparticles (NPs) can be correlated with the particle size and composition, though significant differences in comparison with the bulk can be expected based on theoretical calculations. ${ }^{1}$ The asdefined solid solutions or segregated Au-Fe NPs are very tedious to produce using chemical co-precipitation and reversed micelle methods. ${ }^{6}$ Laser ablation in liquid (LAL) ${ }^{7}$ is a viable alternative, producing ligand free nanoparticles on a gram scale. ${ }^{8}$ To date some research in the area of laser-generated gold rich Au-Fe alloy NPs has been conducted aiming at control over optical and magnetic properties in correlation with the composition and the surrounding media. Metastable alloys with different mixing grades ${ }^{9}$ were synthesized, with clear dependency on the molar ratio $^{10}$ and surrounding liquids. ${ }^{11}$ Even though the Au-Fe system has been subjected to a number of experimental investigations a deeper understand-

\footnotetext{
${ }^{a}$ Technical Chemistry I and Center for Nanointegration Duisburg-Essen (CENIDE), University Duisburg-Essen, Universitätstr. 7, 45141 Essen, Germany

${ }^{b}$ Faculty of Engineering CAU Kiel, Institute for Materials Science, Synthesis and Real Structure, Kaiserstraße 2, 24143 Kiel, Germany

${ }^{c}$ Department of Inorganic Chemistry, University Duisburg-Essen, Universitätstr. 7, 45141 Essen, Germany.E-mail: stephan.barcikowski@uni-due.de

$\dagger$ Electronic supplementary information (ESI) available. See DOI: 10.1039/ c8nr03962c
}

ing of the complex formation mechanism in correlation with the target composition and the particle diameter is still lacking particularly for iron-rich systems where segregated structures are anticipated based on the bulk phase diagram.

Herein we report that in $\mathrm{Au}-\mathrm{Fe}$ NPs formed by $\mathrm{LAL}$ the emergence of a core shell (CS) structure is favored for NPs with a diameter exceeding $10 \mathrm{~nm}$, Fe molar fractions in the target $>35 \%$ and deviating crystal structures (Au-face centered cubic (FCC) \& Fe-body-centered cubic (BCC)). On the other hand, disordered solid solution (SS) nanoparticles with the Fe-FCC structure and number mean diameters $<10 \mathrm{~nm}$ always form independent of the Fe content. Au-Fe NPs were synthesized using LAL in acetone (see the ESI for details $\dagger$ ) from bulk targets with varied $\mathrm{Fe}$ : Au ratios. High resolution imaging by scanning transmission electron microscopy (TEM) in combination with energy-dispersive X-ray spectroscopy (EDX) line scans revealed the formation of two internal phase structures, a disordered solid solution and a FeAu@AuFe core-shell structure clearly distinguishable by the presence/absence of a defined phase boundary detected in TEM and EDX (Fig. 1 and $\mathrm{S} 1 \dagger)$. In consecutive experiments we aimed at elucidating to what extent the emergence of these structures was correlated with the target composition, the particle diameter and the overall crystal structure determined by X-ray powder diffraction (XRD).
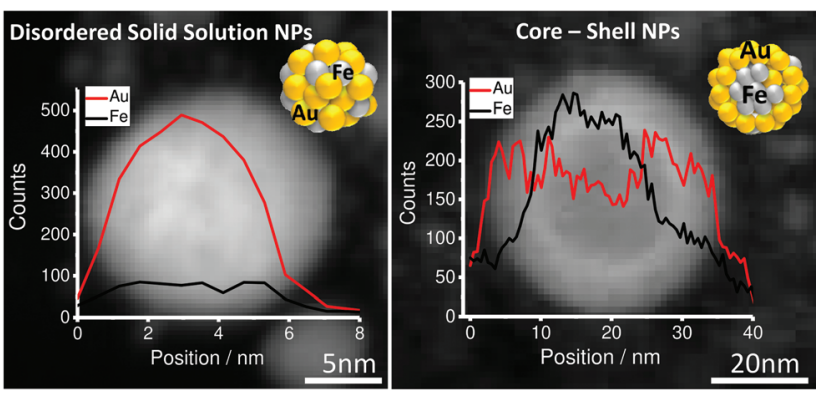

Fig. 1 EDX composition analysis for Au-Fe LAL generated NPs in acetone (disordered solid solution SS [left] and core-shell CS [right]). 
Notably, for iron rich NPs (Fe $>35 \%$ ), 2 fractions of NPs with number mean diameters $>$ and $<10 \mathrm{~nm}$ were detected (Fig. 2a and $\mathrm{S} 2 \dagger$ ). Bimodal size distributions were expected as previously shown, based on different ablation mechanisms. ${ }^{8}$ In contrast, from $\mathrm{Au}$ rich targets ( $\mathrm{Fe}<35 \%)$ monomodal particle size distributions with number mean diameters $<10 \mathrm{~nm}$ were generated. In this context it should be noted that when discussing number mean diameters, derived from fitting particle size distributions with a log-normal function, the presence of low fractions of particles $>10 \mathrm{~nm}$ cannot be excluded in these samples. The overall reduced abundance of larger particles in gold-rich alloy NPs may be explained by size quenching caused by specific solvent-surface interactions between gold-rich surfaces and acetone. ${ }^{12}$

Based on this we deduced a clear particle diameter/Fe\%structure dependency. Our analysis revealed that all particles with number mean diameters $<10 \mathrm{~nm}$ had a disordered solid solution structure, while the core-shell was only found for NPs $>10 \mathrm{~nm}$. This trend was quantified by determining the volumeweighted CS NPs yield (Fig. 2a). It may be concluded that phase segregation (CS-formation) is only observed for NPs with diameters $>10 \mathrm{~nm}$ and Fe molar fractions $>35 \%$. Additionally, disordered SS forms independent of the target composition.
Thermodynamic calculations, described in more detail elsewhere by Chattopadhyay et al., ${ }^{13}$ were employed to predict how the most favored ultrastructure (SS or CS NPs) depends on the particle diameter (Fig. 2c). In this model surface free energies for CS and SS morphologies at the predefined diameter and composition are calculated, indicating the thermodynamically most stable structure by minimum Gibbs energy, confirmed by Wulff construction. ${ }^{14}$ Even though a slight dependency of the free energy on the composition was observed, the impact of the particle diameter was much more pronounced. The results clearly show that NPs with diameters $<7 \mathrm{~nm}$ would be thermodynamically more stable as SS NPs independent of the Fe molar ratio. In the case of NPs with diameters $>7 \mathrm{~nm}$, phase segregation and hence the emergence of CS NPs should be preferred (Fig. 2c). These calculations confirm our experimental findings, showing CS structures only for mean particle diameters $>10 \mathrm{~nm}$ (Fig. 2a). The correlation between the diameter and CS yield was further verified by investigation of two lasergenerated colloids with deviating diameter distributions but identical target compositions $\left(\mathrm{Au}_{50} \mathrm{Fe}_{50}\right)$ (Fig. 2b). The results are fully consistent with our expectations, the number of generated CS increases with increasing NP size. Even though the CS yield increased with increasing Fe molar fraction and
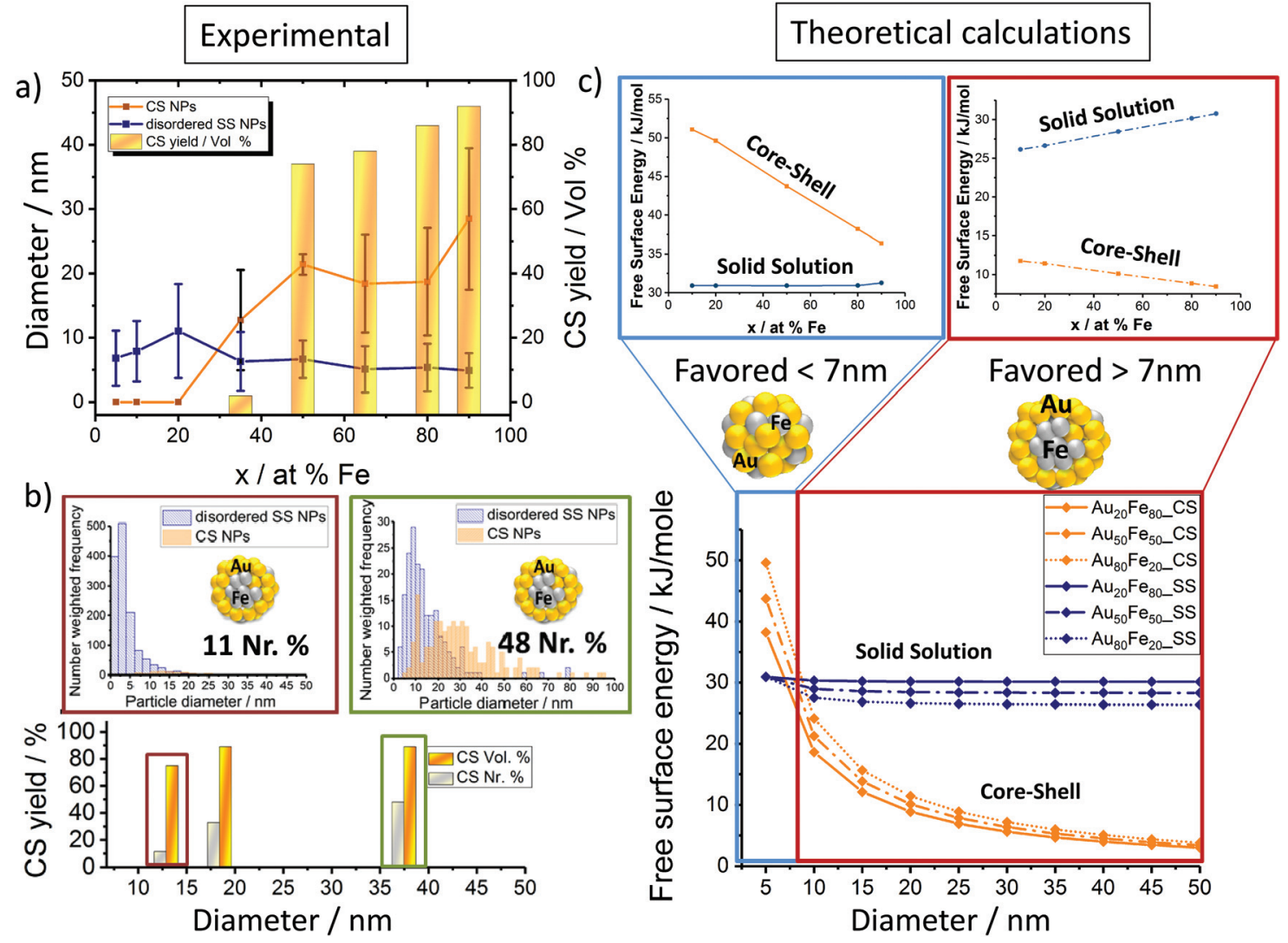

Fig. 2 Factors determining the disordered solid solution versus the core-shell NP yield; (a) nanoparticle size-target composition dependency (disordered SS \& CS NPs) for Au-Fe NPs generated via LAL in acetone. (b) Influence on the CS yield\% based on the average size of Au-Fe NPs for both volume (vol\%) and number ratio ( $\mathrm{Nr} \%$ ). (c) Theoretical calculations are based on the Chattopaghyay model. ${ }^{13}$ 
diameter, the disordered SS alloy NPs are always present independent of the target composition. This is surprising as in accordance with the Hume-Rothery rule,$^{15}$ miscibility of Au and Fe defines the system as mostly immiscible (bulk solubility at room temperature: $3 \% \mathrm{Au}$ in $\mathrm{Fe}$ and $0.3 \% \mathrm{Au}$ in $\mathrm{Fe})^{5}$ because of the differences in the lattice parameters $\left(\mathrm{Au}_{\mathrm{FCC}} 4.076 \AA\right.$; $\mathrm{Fe}_{\mathrm{BCC}} 2.866 \AA$ ) and in the surface energies ( $\mathrm{Au} 1.5 \mathrm{~J} \mathrm{~m}^{-2} ; \mathrm{Fe}$ $\left.2.4 \mathrm{~J} \mathrm{~m}^{-2}\right) \cdot{ }^{16,17}$ Based on this, the segregated phase (CS) should be the only thermodynamically stable phase. To examine this phenomenon in more detail, we determined the crystal structure of the generated Au-Fe NPs by X-ray powder diffraction (XRD) including Rietveld refinement and the calculation of lattice parameters for FCC and BCC phases (Fig. 3 and Fig. S3 $\dagger$ ). The analysis revealed that for target compositions $>65 \%$ a BCC iron phase was dominant in the NPs, which basically coincides with the occurrence of CS phase structures in transmission electron microscopy. In this context it should be noted that the lattice constants of this phase are slightly elevated compared to bulk $\mathrm{Fe}$, which points at partial substitution of iron with $\mathrm{Au}$ within the particle's core. In addition, a $\mathrm{Au}-\mathrm{Fe}$ FCC structure is detectable over the whole composition range, which can be clearly attributed to the disordered SS NPs in gold rich samples. In iron rich samples, however, disordered SS NPs and gold rich shells cannot be differentiated based on XRD. This Au-FCC phase exhibits a reduction in lattice parameters from 4.1 to $3.95 \AA$ while increasing the iron molar fraction up to $65 \%$, which evidences alloy formation and substitutions by iron atoms in the FCC-Au lattice. As the substitutions are significantly higher than those predicted by the bulk phase diagram (Fig. 4), the formation of a metastable phase is verified. For iron contents $>65 \%$, the substitution by Fe within the FCC-Au lattice decreases due to competition between the BCC/ FCC phase as predicted by Baricco. ${ }^{18}$ In general, the FCC Au-Fe phase exhibits the strongest negative deviations from Vegard's law at a composition around $50: 50$. Similar observations were also made in $\mathrm{Ag}-\mathrm{Au}$ nanoparticles produced by the same method. ${ }^{19}$ Finally, the mass fractions of FCC/BCC were calcu-

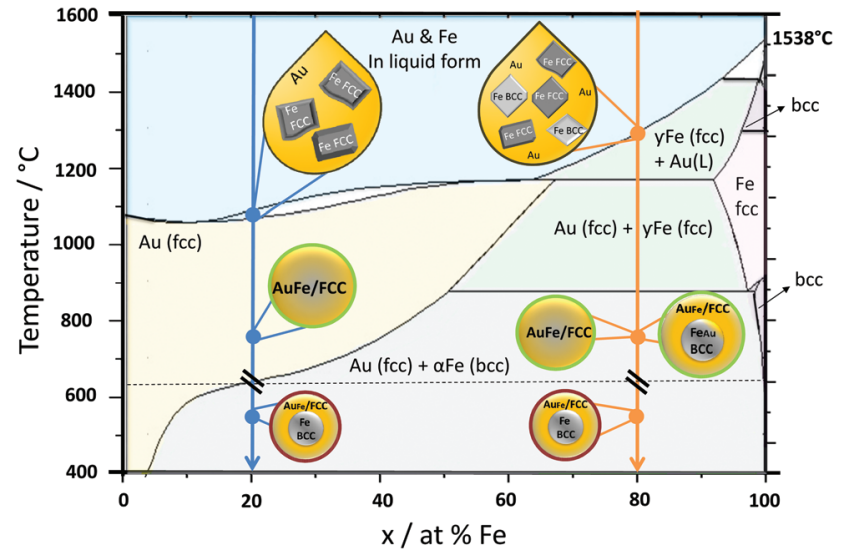

Fig. 4 Formation mechanism for Au-Fe nanoparticles generated by LAL. Cool down arrows showing two representative phases, $\mathrm{Au}_{20} \mathrm{Fe}_{80}$ (orange line) and $\mathrm{Au}_{80} \mathrm{Fe}_{20}$ (blue line). Green circles indicate the metastable product found as a product of our synthesis, while red circles show the product which would be most stable according to the bulk phase diagram but which is NOT synthesized in our process. The Au-Fe phase diagram redrawn from Ref. 14.

lated from the Rietveld refined peaks (Fig. 3). Here we can clearly observe that an increase in the BCC fraction also goes along with a more pronounced CS formation (compare Fig. 2a). These data show that next to the size restrictions, the NP crystal structure correlates with the phase segregation. However, one peculiarity was found for the $\mathrm{Au}_{50} \mathrm{Fe}_{50}$ as well as to a smaller extent for the $\mathrm{Au}_{65} \mathrm{Fe}_{35}$ composition. Here we clearly verified the formation of CS structures by TEM, while on the other hand no BCC-iron was found. These findings may indicate that CS structures may also form based on two FCC alloy structures (gold-rich $\mathrm{Au}-\mathrm{Fe}$ shell around the iron-rich $\mathrm{Fe}-\mathrm{Au}$ core) with distinguishable compositions. Here AuFe@AuFe is probably formed. The presence of up to three distinguishable FCC structures in XRD (FCC 1, FCC 2 and FCC 3 in Fig. 3) seems to point in this direction. In this context the iron-rich FCC (lower
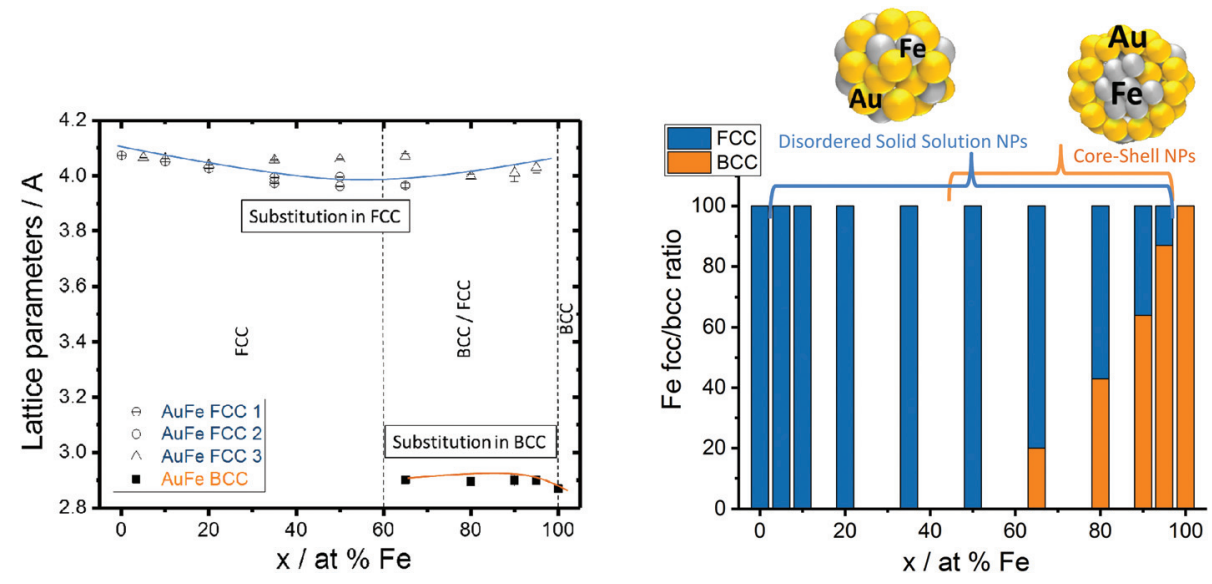

Fig. 3 Au-Fe lattice parameters (FCC 1, FCC 2, and FCC 3 represent alloy structures with different substitution grades) and weighted phase ratios between $\mathrm{BCC} / \mathrm{FCC}$ structures measured by XRD. 
lattice parameter, FCC 1 and FCC 2) could come from the core and the gold-rich FCC (higher lattice parameter, FCC 3) could originate from the shell. However, this phenomenon cannot be comprehensively explained based on these data.

As we could clearly verify that the LAL-generated iron-rich $\mathrm{Au}-\mathrm{Fe}$ NPs are metastable structures with alloy cores and alloy shells, in this final paragraph we aim to elucidate their formation mechanism during LAL based on the bulk $\mathrm{Au}-\mathrm{Fe}$ phase diagram (Fig. 4). At the first stage both $\mathrm{Au}$ and Fe are rapidly cooled down from the liquid state. Gold would stay longer in this state as its melting temperature is lower (Au $1064{ }^{\circ} \mathrm{C}$; Fe $1538{ }^{\circ} \mathrm{C}$ ). ${ }^{18}$ Therefore, at the liquidus line interface, solid Fe would be surrounded by liquid Au. In the case of $\mathrm{Fe}$ rich systems, e.g. $\mathrm{Au}_{20} \mathrm{Fe}_{80}$, Fe can crystallize as either BCC or FCC. Hence both structures will be present upon solidification of the entire phase and will shape the final structure, containing BCC iron rich cores with minimal Au substitution as well as gold rich FCC phases, either as isolated particles or as gold-rich alloy shells. Metastable states, in this case, are indicated by higher substitution of $\mathrm{Fe}$ in $\mathrm{Au}$ and $\mathrm{Au}$ in $\mathrm{Fe}$ in contrast to thermodynamically-favored phases at room temperature. On the other hand, for $\mathrm{Au}$ rich alloys, e.g. $\mathrm{Au}_{80} \mathrm{Fe}_{20}$, only FCC iron can form upon crystallization. As the phase transformation from FCC to BCC may be kinetically hindered due to the fast cooling inherent to the LAL process, and FCC formation may require a lower driving force of formation (as the matrix is also FCC), ${ }^{5}$ therefore only FCC Au-Fe alloy NPs emerge and no BCC iron is found.

In conclusion, the final internal phase structure (ultrastructure) of Au-Fe NPs generated by LAL in acetone is critically affected by the target composition and the particle diameter. The formation of CS is favored in iron-rich targets (Fe mol\% $>35$ ), for particles $>10 \mathrm{~nm}$ and results in BCC iron crystal structures. We could conclusively prove that LAL generates nonequilibrium alloy materials containing FeAu@AuFe core-shell structures where the core and shell are both alloys. This underlines the fundamental significance of this work in understanding the formation of disordered solid solution alloys and segregated nano structures in the $\mathrm{Au}-\mathrm{Fe}$ system, which may be initial steps towards a nanoscale-phase diagram with particle size as a third axis next to composition. Furthermore, this work may also give access to novel metastable $\mathrm{Au}-\mathrm{Fe}$ alloy nanomaterials with potential future applicability in catalysis and biomedicine.

\section{Conflicts of interest}

The authors declare no conflict of interest.

\section{Acknowledgements}

We acknowledge financial support from DFG (BA 3580/18-1 and KI 1263/15-1).

\section{References}

1 F. Calvo, Nanoalloys: From Fundamentals to Emergent Applications, Elsevier, 2013.

2 A. H. Lu, E. L. Salabas and F. Schuth, Angew. Chem., Int. Ed. Engl., 2007, 46, 1222-1244.

3 I. Vassalini, L. Borgese, M. Mariz, S. Polizzi, G. Aquilanti, P. Ghigna, A. Sartorel, V. Amendola and I. Alessandri, Angew. Chem., Int. Ed. Engl., 2017, 56, 6589-6593.

4 L. M. Liz-Marzan, Langmuir, 2006, 22, 32-41.

5 D. Favez, J. D. Wagniere and M. Rappaz, Acta Mater., 2010, 58, 1016-1025.

6 I. Sharifi, H. Shokrollahi, M. M. Doroodmand and R. Safi, J. Magn. Magn. Mater., 2012, 324, 1854-1861.

7 D. Zhang, B. Gokce and S. Barcikowski, Chem. Rev., 2017, 117, 3990-4103.

8 R. Streubel, S. Barcikowski and B. Gokce, Opt. Lett., 2016, 41, 1486-1489.

9 V. Amendola, S. Scaramuzza, L. Litti, M. Meneghetti, G. Zuccolotto, A. Rosato, E. Nicolato, P. Marzola, G. Fracasso, C. Anselmi, M. Pinto and M. Colombatti, PubMed, 2014, 10, 2476-2486.

10 Z. Swiatkowska-Warkocka, A. Pyatenko, K. Koga, K. Kawaguchi, H. Wang and N. Koshizaki, J. Phys. Chem. C, 2017, 121, 8177-8187.

11 P. Wagener, J. Jakobi, C. Rehbock, V. S. Chakravadhanula, C. Thede, U. Wiedwald, M. Bartsch, L. Kienle and S. Barcikowski, Sci. Rep., 2016, 6, 23352.

12 V. Amendola and M. Meneghetti, Phys. Chem. Chem. Phys., 2009, 11, 3805-3821.

13 C. Srivastava, S. Chithra, K. D. Malviya, S. K. Sinha and K. Chattopadhyay, Acta Mater., 2011, 59, 6501-6509.

14 G. D. Barmparis, Z. Lodziana, N. Lopez and I. N. Remediakis, Beilstein J. Nanotechnol., 2015, 6, 361-368.

15 U. Mizutani, Hume-Rothery Rules for structural Complex Alloy Phases, 2011.

16 C. Langlois, P. Benzo, R. Arenal, M. Benoit, J. Nicolai, N. Combe, A. Ponchet and M. J. Casanove, Nano Lett., 2015, 15, 5075-5080.

17 F. Calvo, N. Combe, J. Morillo and M. Benoit, J. Phys. Chem. C, 2017, 121, 4680-4691.

18 M. Baricco, METALLURGIA FISICA, 2004.

19 O. Prymak, J. Jakobi, C. Rehbock, M. Epple and S. Barcikowski, Mater. Chem. Phys., 2018, 207, 442-450. 